\title{
RICONOSCIMENTO ED ESECUZIONE DELLE SENTENZE STRANIERE IN TURCHIA*
}

\author{
Yüksel ERSOY *
}

\section{IN GENERALE}

Una sentenza straniera è una sentenza pronunciata da una autorità giudiziaria di un pacse stranicro.

La materia del riconoscimento e dell'esecuzione di sentenze dei tribunali ordinari ed arbitrali che era in precedenza disciplinata da una Sezione del Codice di Procedura Civile, è ora regolata nella II. Sezione intitolata "Esecuzione e Riconoscimento delle Sentenze e Lodi Arbitrali Stranieri" dagli articoli 39-45 della legge speciale n. 2675, intitolata "Legge sul Diritto Internazionale Privato e sul Diritto di Procedura Internazionale" (LDPPI) e emanata il 20 Maggio 1982. Negli articoli 34-42 sono previste le modalità di riconoscimento ed esecuzione delle sentenze straniere, mentre negli articoli $43-45$ si regolano le stesse modalità in relazione alle sentenze arbitrali straniere.

Nella LDPPI, a differenza di altri sistemi che prevedono il riconoscimento come prerequisito della esecutorietà delle sentenze straniere, le due istituzioni sono regolate separatamente, anche se l'accettazione della esecutorietà comporta anche il riconoscimento di una determinata sentenza. Come cerchero di spiegare piu avanti, nel sistema della LDPPI, oltre alla decisione di esecutorietà che richiede l'esistenza di determinate condizioni, vi è anche la possibilità di dichiarazione del riconoscimento soltanto (e cioè senza possibilità di esecuzione) nel caso di esistenza di parte delle condizioni.

\footnotetext{
*Questa relazione è stata presentata al 6 Convegno Internazionale dell'Associazione Giruristi di Lingua Italiana svoltosi a Porto Cervo dal 23 al 26 Settembre 1993.

**Yuksel Ersoy è professore di diritto penale alla Facolta di Scienze Politiche dell'Universtià di Ankara.
} 


\section{RICONOSCIMENTO ED ESECUZIONE DELLE SENTENZE STRANIERE}

Secondo l'articolo 34, è indispensabile ottenere la sentenza di esecutorietà da un tribunale turco per poter eseguire una sentenza pronunciata da un tribunale straniero in materia civile.

Le condizioni previste affinchè il tribunale turco possa dichiarare l'esecutorietè della sentenza straniera sono elencate nell'articolo 38.

\section{Richiesta della parte interessata}

La decisione di esecutorietà si pronuncia in base ad una domanda (art. 36) mediante citazione davanti alla corte compenente, posta dalla parte interessata con la presentazione della documentazione menzionata all'articolo 38.

Nella domanda lo scopo di ottenere l'esecuzione dev essere indicato chiaramente. Pertanto, se la domanda è presentata, per esempio, come azione di risarcimento sulla base di una sentenza straniera, e poi s'intende cambiarla in una richiesta di dichiarazione di esecutorietà, questo sarà possibile soltanto o con la riforma dell'azione o con il consenso del convenuto.

\section{Necessità di sentenza straniera in materia civile}

La sentenza di cui s'intende ottenere l'ammisssione di esecutorietà deve essere emessa in materia civile. Non $\mathrm{e}$, quindi, possibile chiedere ed ottenere la dichiarazione di esecutorietà delle sentenze pronunciate in materia penale.

A tale scopo, è da precisare perd, che non è importante se il tribunale che emette la sentenza sia civile o penale o che le parti siano persone di diritto civile o istituzioni statali, ma è importante che la controversia sia originata da una relazione di dirito civile. Pertanto, anche $i$ diritti personali contenuti in una sentenza penale possono essere eseguiti in base ad una decisione di esecutorietà (art. 34 comma 2).

\section{Necessità di cosa giudicata}

La sentenza straniera per poter essere dichiarata eseguibile deve essere passata in giudicato. La definitività di una sentenza interessa, naturalmente, sia l'aspetto formale che sostanziale.

Nel sistema della procedura civile, di regola, le sentenze acquistano definitività formale e sostanziale nello stesso tempo del compimento dell'iter procedurale, con l'eccezione del non-contenzioso dove si può parlare soltanto della definitività formale. La dottrina in generale ritiene sufficiente la definitività formale, partendo dalla lettera dell'articolo 34.

Un'altra considerazione da fare in merito alla definitività della sentenza è quella relativa all'accertamento del sistema legale secondo il quale essa va valutata. L'articolo 34 
prevede che tale valutazione venga fatta in base al sistema legale del paese dove e pronunciata la sentenza.

\section{Decisione di esecuzione (Exaequatur)}

L'articolo 34 prevede la necessità di ottenere una decisione di esecuzione da un tribunale turco che accerta l'esistenza delle condizioni richieste dalla legge. La decisione della corte viene chiamata exaequator. ${ }^{1}$

\subsection{La Corte compenente}

Secondo l'aricolo 35, la conte competente ad emeuere la decisione dell'exaequator è la corte del domicilio in Turchia della persona (la controparte) contro la quale s'intende eseguire la sentenza. Se la controparte non ha un domicilio in Turchia, la corte competente è quella della residenza della controparte e se anche questa manca, una delle corti di Ankara, Istanbul e lzmir avrà la competenza. ${ }^{2}$

La domanda dcve essere presentata con aggiunta di tante copie quanto è il numero delle persone costituenti la controparte.

\subsection{Contenuto della domanda}

Nella domanda devono essere incluse i dati seguenti:

4.2.1. I nomi, cognomi, indirizzi delle parti e dei loro rappresentanti.

4.2.2. Lo Stato dove è stata pronunciata la sentenza, la corte che ha pronunciata la sentenza, la data ed il numero della sentenza insieme ad un riassunto della sentenza.

4.2.3. Indicazione della parte di sentenza di cui si chiede l'efficacia, se si intende una esecuzione parziale.

\subsection{Documenti da includere}

I seguenti documenti devono essere aggiunti alla domanda secondo l'articolo 37:

4.3.1. Copia originale certificata della sentenza e la sua truduzione certificata.

4.3.2. Dichiarazione certificata del passaggio in giudicato della sentenze e la sua traduzione centificata.

${ }^{1}$ Çelikel, Prof. Dr. Aysel, Milletlerarası Ozel Hukuk, (Diritto Internazionale Privato), Istanbul; 1987, 2. ed, p. 294-298.

${ }^{2}$ Kuru, Prof. Dr. Baki, Hukuk Muhakemeleri Usulü. (Diritto di Procedura Civile), Ankara, 1984, 4. ed., v. IV, p. 3927-3932. 
La necessità di presentazione di documenti sopra elencati è confermata anche dalla Corte di Cassazione. ${ }^{3}$

\subsection{Condizioni per la decisione}

L'articolo 38 fa un elenco delle condizioni necessarie per il riconoscimento dell'efficacia di una sentenza straniera.

Queste condizioni devono essere valutate insieme ad alture due condizioni generali già menzionate, e cioe la sentenza in materia civile e la sentenza passata in giudicato ed il giudice è tenuto a dichiarare l'esecutorietà quando accerta l'esistenza di tutte le condizioni. ${ }^{4}$

\subsubsection{La Reciprocità}

\subsubsection{Contenuto}

Una delle condizioni da accertare è la reciprocità in materia di esecuzione tra la Turchia e lo Stato nel quale è stata pronunciata la sentenza. La Cassazione considera questa condizione come conditio sine qua non ${ }^{5}$ per la dichiarazione della esecutorietà. La reciprocità esiste quando vi è un accordo o una convenzione o una norma di legge oppure una pratica di fatto che rende possibile l'esecuzione di una sentenza pronunciata da un tribunale turco nello Stato in questione. L'ammissione della reciprocità sia legale che di fatto è confermata anche dalla giurisprudenza della Cassazione. ${ }^{6}$

La reciprocità deve essistere al tempo della domanda di dichiarazione di esecutorietà $\mathrm{e}$ non al momento del passaggio in giudicato della sentenza straniera. Il giudice accerta l'esistenza o meno della reciprocità, d'ufficio.

- Le condizioni di reciprocità si differenziano da un paese all'altro. Essa può essere ritenuta esistente tra la Turchia ed un altro Stato, quando le condizioni previste per la dichiarazione di esecutorietà di una sentenza di un tribunale turco non sono più pesanti di quelle previste nella legislazione turca.

In particolare, la reciprocità non sarebbe ammessa nel caso in cui una delle condizioni previste nella legislazione straniera è il riesame del merito della sentenza del tribunale turco.?

${ }^{3}$ Corte di Cassazione, 2. HD, E 1986/5862, K. 1986/6076, T.17.6.1986 (YKD 1987-2/154).

${ }^{4}$ Kuru, op. cit., p. 3922.

${ }^{5}$ Corte di Cassazione, HGK, E. 1990/13-3, K. 1990/347. T. 13.6.1990 (YKD 1990-9/1282); 13. HD, E. 1990/4485, K. 1990/8676, T. 13.12.1990 (YKD 1991-5/29).

${ }^{6}$ Çelikel, op. cit., p. 301.

${ }^{7}$ Nomer, Prof. Dr. Ergin, Devletler Hususi Hukuku, (Diritto Intemazionale Privato), Istanbul, 1990, p. 464-465. 


\subsubsection{Gli accordi}

$\mathrm{Ci}$ sono accordi internazionali multilaterali e bilaterali con effetto sul riconoscimento ed esecuzione delle sentenze straniere di cui la Turchia è parte.

Gli accordi multilaterali sono i seguenti:

a) Convenzione sul Riconoscimento ed Esecuzione di Sentenze concernenti l'Obbligo di Provvedere Alimenti ai Bambini (pubblicata sulla Gazzetta Ufficiale dell'11 Gennaio 1973).

b) Convenzione sul Riconoscimento e Esecuzione di Sentenze concernenti Crediti di Alimenti (pubblicata sulla Gazzetta Ufficiale del 16 Febbraio 1983).

c) Convenzione concernente la Riscossione di Crediti di Alimentí in Paesi Stranieri (pubblicata sulla Gazzetta Ufficiale del 18 Marzo 1971).

d) Convenzione sul riconoscimento delle Sentenze concernenti Relazioni Matrimoniali (pubblicata sulla Gazzetta Ufficiale del 14 Settembre 1975).

e) Convenzione sul Trasporto di Persone e Beni per Ferrovie (pubblicata sulla Gazzeua Ufficiale del 3 Marzo 1965 e del 4 Agosto 1969).

Gli accordi bilaterali sono quelli firmati con l'Italia (Gazzetta Ufficiale del 3 Marzo 1929), l'Austria (Gazzetua Ufficiale del 4 Agosto 1931), la Rumania (Gazzetta Ufficiale del 3 Marzo 1972) e la Tunisia (Gazzeta Ufficiale del 11 Settembre 1984).

\subsubsection{Ordine pubblico}

La sentenza straniera non deve contenere disposizioni contrarie all'ordine pubblico turco. Vi è contrarietà quando il contenuto della sentenza straniera ha un elemento in chiara contraddizione con l'ordine pubblico turco. ${ }^{8}$

L'applicazione dei principî di un sistema legale straniero di per sè non costituisce. per il giudice turco, un motivo di rigetto. ${ }^{9}$

Comunque, si potrà parlare di contrarietà all'ordine pubblico, se la sentenza straniera è basata su principî che sono in contrasto con i principî essenziali del sistema legale turco, sia sostanziali che formali.

Il diritto straniero avente un elemento di discriminazione tra i sessi o di esclusione o eccessiva limitazione del diritto di difesa costituirebbe esempio di chiaro contrasto rispettivamente con il diritto sostanziale e formale.

Anche gli accordi bilaterali firmati dalla Turchia e in particolare l'accordo con l'italia ammette espressamente il contrasto con l'ordine pubblico quando esistono due senteze

${ }^{8}$ Nomer, op. cit., p. 467.

9Nomer, ibid. 
contrastanti, una straniera ed una turca. ${ }^{10}$ La Cassazione considera tale situazione come motivo di revisione della sentenza. ${ }^{11}$

Secondo il Professor Çelikel, l'esistenza di una causa pendente in Turchia sullo stesso oggetto su cui esiste una sentenza straniera definitiva dovrebbe comportare il rigetto della richiesta di riconoscimento ed esecuzione per l'effetto degli accordi bilaterali. ${ }^{12}$

\subsubsection{Competenza esclusiva dei tribunali turchi}

La sentenza straniera non può essere dichiarata eseguibile, se una materia è di competenza esclusiva dei tribunali turchi (art. 38/b). Ci sono varie opinioni per quanto concerne il concetto della "compenenza esclusiva", ma un punto indiscusso è in relazione all'appartenenza a questa categoria della competenza dei tribunali turchi sulle cause relative ai beni immobiliari in Turchia.

Secondo il Professor Nomer, il concetto della competenza esclusiva che impedisce la dichiarazione di esecutorietà è dịfferente dal concetto di competenza esclusiva nel diritto interno. Affinchè una norma riguardante la competenza possa impedire la dichiarazione di esecutorietà, essa dovrebbe essere rivolta alla valutazione della materia da parte, solamente, di tribunali turchi. ${ }^{13}$

Anche nel caso in cui la competenza è determinata con l'accordo tra le parti, la regola della competenza esclusiva potrebbe ancora avere effetto di impedire la dichiarazione di esecutorietà se la sentenza è pronunciata in un'area di diritto dove $\mathrm{i}$ tribunali turchi hanno competenza esclusiva. ${ }^{14}$

\subsubsection{Rispetto del diritto di difesa}

La condizione del rispetto del diritto di difesa (art. 38/) è una garanzia di giusto processo (fair trial) a favore della parte contro la quale si intende ottenere l'esecuzione.

Le situazioni che non rientrano nell'ambito di questo paragrafo possono essere considerate contemplate dal principio dell'ordine pubblico, poichè qualsiasi violazione del diritto di difesa può essere considerata in contrasto con tale principio. ${ }^{15}$

La mancata osservanza del diritto di difesa, per poter essere presa in considerazione da parte del tribunale turco competente, deve essere avanzata dalla parte contro la quale si chiede la dichiarazione di esecutorietà.

\footnotetext{
${ }^{10}$ Çelikel, op. cit., p. 312.

${ }^{11}$ Corte di Cassazione, 2. HD, E. 1984/9293, K. 1984/9484, T. 15.11 .1984 (YKD 1985-2/185).

${ }^{12}$ Çelikel, op. cit., p. 313

13 Nomer, op. cit., p. 466.

${ }^{14}$ Nomer, op. cit., p. 467.

${ }^{15}$ Çelikel, op. cit,, p. 314 .
} 


\subsubsection{Stati personali dei turchi}

Un'altra condizione per la dichiarazione di esecutorietà è l'applicazione del diritto indicato dalle norme sui conflitti di legge vigenti in Turchia nelle sentenze straniere concernenti gli stati personali dei turchi. La corte compentente non può dichiarare l'esecutorietà, se tali norme non sono rispettate ed il convenuto di cittadinanza turca si oppone alla esecuzione.

\section{Ricorso}

Nel caso dell'accoglimento della domanda di riconoscimento ed esecuzione, il convenuto ha il diritto di fare ricorso in Cassazione secondo la norme procedurali generali in base all'articolo 41 comma 2 della LDPPI. Il ricorso sospende l'esecuzione della sentenza.

\section{RICONOSCIMENTO DELLE SENTENZE STRANIERE}

\section{1- In generale}

Come accennato in precedenza, nel sistema della LDPPI, oltre alla dichiarazione di esecutorietà che implicitamente presuppone il riconoscimento di una sentenza straniera, vi è anche la figura del riconoscimento senza diritto all'esecuzione.

In questo senso, una sentenza straniera riconosciuta contiuisce prova decisiva o cosa giudicata secondo l'articolo 42.

La decisione del riconoscimento d̀ pronunciata dalla corte competente indicata nell'articolo 35 (v. prg. 4. 1. sopra), la quale accerta se esistono le condizioni necessarie che sono identiche a quelle del caso di esecuzione con l'eccezione della reciprocita (art. 38/a) e del rispetto del dirito della difesa (ar. 38/d).

\section{2- Procedura}

La giurisprudenza considera la procedura per il riconoscimento delle sentenze straniere come azione di accertamento. Pertanto, La Corte Suprema richiede l'esistenza di un interesse giuridico per poter far iniziare questa procedura, ${ }^{16}$ poichè l'esistenza di un interesse giuridico costituisce conditio sine qua non di una tale azione nel diritto di procedura civile ed il giudice è tenuto a verificarne l'esistenza d'ufficio. L'interesse giuridico non esiste se il beneficio aspettato dal riconoscimento può essere ouenuto con un'azione per la dichiarazione di esecutorieta. L'azione di acceramento non ha esecutorietà, ${ }^{17}$ poichè non contiene nessun ordine di adempimento. ${ }^{18}$

${ }^{16}$ Corte di Cassazione, 4. HD, E. 1985/5537, K. 1985/7507, T. 30.9 .1985 (YKD 1986-1/39). 17 Kuru, op. cit., p. 247-251.

${ }^{18}$ Corte di Cassazione, 13. HD, E. 1989/1221, K. 1989/4636, T. 30.6.1989 (YKD 1990$7 / 1029$ ). 


\section{Condizioni di riconoscimento}

In conseguenza del riferimento fatto dall'articolo 42 all'articolo 38 , le condizioni necessarie per la dichiarazione di esecutorietà sono anche valide per la decisione di riconoscimento, eccezione fatta, come già spiegato, per i paragrafi (a) e (d) concementi la reciprocità ed il rispetto del diritto della difesa.

Riconoscimento delle sentenze straniere pronunciate nella giurisdizione volontaria è ottenuto con la stessa procedura (LDPPI art. 42 comma 2 e 3).

\section{IV- RICONOSCIMENTO ED ESECUZIONE DELLE SENTENZE ARBITRALI}

\section{1- In generale}

Nella legislazione turca vi sono due fonti, una interna l'altra internazionale per il riconoscimento ed esecuzione delle sentenze arbitrali stranicre. Quella interna è sempre la LDPPI che dedica a questo argomento gli articoli 43-45, mentre nel campo internazionale la Turchia ha aderito alla "New York Convention on the Recognition And Enforcement of Foreign Arbitral Awards" (La Convenzione).

La Turchia ha aderito alla Convenzione ratificandola con la legge dell'8 Maggio 1991 pubblicata sulla Gazzetta Ufficiale il 25 Settembre 1991 e mettendo la riserva della condizione della reciprocità in base all'articolo I.3 della Convenzione stessa.

Secondo la Costituzione, gli accordi internazionali approvati acquistano potere de legge in vigore nel territorio dello Stato. Nel caso di conflitto tra una norma di legge e la norma di un accordo internazionale successivamente adottato, a mio parere, dovrebbe valere la norma dell'accordo in base ai principî di lex posteriori e pacta sunt servanda.

Le norme contenute nella LDPPI per quanto concerne le sentenze arbitrali straniere riguardano soltanto la loro esecuzione, mentre, a differenza delle sentenze straniere, non contemplano separatamente l'ipotesi del riconoscimento. Comunque, la dottrina è unanime nell'accettare la possibilità di applicare la stessa procedura per ambedue i casi. ${ }^{19}$

Nella LDPPI il titolo della Sezione II è "Esecuzione e Riconoscimento delle Sentenze dei Tribunali e Tribunali Arbitrali Stranieri", pertanto sembra che il Legislatore non abbia inteso un trattamento differente per $i$ lodi arbitrali.

Nella Convenzione, anche, si osserva l'uso di ambedue i termini sia nel titolo che nel testo, mentre non si ha una norma distinta per il caso di riconoscimento.

${ }^{19}$ Nomer, op. cit. p. $490-491$. 
Secondo la Convenzione e le norme di legge, il riconoscimento si considera come "conseguenza naturale" della dichiarazione di esecutorietà e dei principî di argumentum $a$ fortiori ${ }^{20}$ e analogia. 21

Quindi, le condizioni del riconoscimento non saranno trattate separatamente, poichè gli stessi principî che regolano la materia dell'esecuzione dei lodi arbitrali saranno validi anche per tale caso.

\section{Condizioni per la dichiarazione di esecutorietà}

Secondo l'articolo 43 della LDPPI è necessaria l'esistenza di tre condizioni per la dichiarazione della esecutorietà delle sentenze arbitrali straniere: esse devono essere definitive, esecutive e straniere.

\subsection{Sentenza arbitrale definitiva}

Come già detto, l'articolo 43 della LDPPI, allo scopo della esecuzione in Turchia, richiede la definitività del lodo arbitrale. Naturalmente, l'esistenza di questo elemento va valutata in base alla legge del luogo dove è stata pronunciata la sentenza.

A differenza del test dell'articolo 43 che richiede "la definitività e l'esecutorietà", il paragrafo (i) dell'articolo 45 parla della definitività o l'esecutorietà della sentenza. Comunque la dottrina non attribuisce importanza a questo uso differente, l'esecutorietà essendo la conseguenza della definitività. ${ }^{22}$

\subsection{Sentenza arbitrale straniera}

La LDPPI non provvede una descrizione del concetto della "sentenza arbitrale straniera". I paragrafi (f), (g) e (i) dell'articolo 45 fanno menzione del principio della "legge cui l'arbitrato è soggetw". La leggè cui si fa riferimento deve intendersi come la legge procedurale accettata dalle parti. Nel caso in cui non vi è un accordo tra le parti, deve essere preso in considerazione il luogo dove il lodo arbitrale viene pronuncialo. Quindi, lodi arbitrali pronunciati nei paesi esteri sono considerati stranieri. ${ }^{23}$ Anche la Cone di Cassazione ha adottato questi due criteri nella valutazione del detto concetto. ${ }^{24}$

20 Unal, Doç. Dr. Şeref, "Yabancı Hakem Kararlarının Tanınması ve Icrası" (Riconoscimento ed esecuzione delle sentenze arbitrali), Avrupa (Cenevre) - New York Sözleşmeleti ve Türk Tahkim Hukuku Sempozyumu, Ankara, 1990, p. 55-56.

${ }^{21}$ Kuru, Prof. Dr. Baki - Yılmaz, Doç. Dr. Ejder; "Türkiye'de Yabancı Hakem Kararlarının Taninmasi" (Riconhoscimento delle sentenze arbitrali straniere in Turchia), II. Tahkim Haftası, Ankara, 1984, p. 193-194.

22 Unal, loc. cit., p. 57.

${ }^{23}$ Nomer, op. cit.. p. 489; Únal, loc. cit., p. 62.

${ }^{24}$ Corte di Cassazione, 15. HD, E. 75/1617, K. 76/1052, T. 10.3.1976; 11. HD. E. 78/5258, K. 78/5916, T. 28.12.1978. 
Come è saputo, anche la Convenzione prevede due criteri nella valutazione del lodo straniero (Articolo I. 1 e Articolo V. 1/a). Pertanto, insieme alla dottrina, si può sostenere che la Legge e la Convenzione siano in armonia tra di loro. ${ }^{25}$

\section{- 2.3. Reciprocità}

La reciprocità d richiesta anche per il riconoscimento ed esecuzione delle sentenze arbitrali straniere in conseguenza del riferimento del paragrafo 2 del secondo comma dell'articolo 44 al paragrafo (a) dell'articolo 38 della LDPPI. Questa disciplina è in armonia con il terzo comma dell'articolo I della Convenzione.

Gli aspetti più importanti sono stati già trattati in relazione alle sentenze straniere. Un particolare punto importante per lodi arbitrali è costituito dalla esistenza di lodi arbitrali cui non può essere attribuita una nazionalità.

Nel caso di una interpretazione restrittiva del principio della reciprocità, lodi arbitrali senza nazionalità non potrebbero essere riconosciuti ed eseguiti in Turchia. Conscia di questa conseguenza, la dottrina tende ad esprimere opinione favorevole ad una considerazione più ampia della reciprocità. ${ }^{26}$

\section{Decisione di riconoscimento}

L'articolo III della Convenzione prevede l'applicazione delle norme procedurali del Territorio dove il riconoscimento e l'esecuzione sono richiesti.

L'articolo V della Convenzione ha adottato l'espressione "autorità compentente" (compentent authority) e tale autorità è la corte di prima istanza nel diritto turco.

É indispensabile l'esistenza di una decisione del tribunale per l'esecuzione di un lodo arbitrale straniero. La decisione del tribunale si scrive in calce alla sentenza arbitrale.

\section{1- Competenza}

La competenza è, in primo luogo, determinato da un eventuale accordo scrituo tra le parti. Nel caso di mancanza di un tale accordo, secondo il secondo comma dell'articolo 42, il tribunale del luogo di domicilio, in mancanza di domicilio, del luogo di residenza, $e$ in mancanza anche di esso, il tribunale del luogo dove si trovano i beni che possono essere oggetto di esecuzione viene considerato competente.

\section{2- Domanda}

Chi richiede il riconoscimento ed esecuzione deve allegare alla sua domanda i documenti precisati nell'articolo 44 della LDPPI conformemente ancha alla Convenzione.

25 Unal, loc. cit., p. 60-63.

${ }^{26}$ Şanlı, Cemal, "Milletlerarası Ticari Tahkimde Hakem Kararlarının Tahkim Yeri Hukukundan Ayrılması ve Bu Kararların 2675 Sayılı Kanun Açısından Tenfizi Sorunu",II.Tahkim Haftas1, Ankara, 1984, p. 183. 


\section{3- Motivi di rigetto}

All'articolo 45 della LDPPI sono elencati i motivi di rigetto della domanda di riconoscimento ed esecuzione.

In questo elenco ci sono nove paragrafi che riguardano i motivi determinanti il rigetto della richiesta mentre nella Convenzione ce ne sono sette. Comunque, questa differenza non comporta una contraddizione tra due testi. Essa è la conseguenza della differenza di sistematica mentre come contenuto ambedue i testi contemplano gli stessi motivi.

Nei paragrafi (d), (e), (f), (g), (h) e (i) l'onere di prova è a carico del convenuto, in conformità anche con il sistema adottato dalla Convenzione. ${ }^{27}$

\section{Ricorso}

Secondo l'articolo 41 della LDPPI, il lodo arbitrale straniero è eseguito come se fosse un lodo arbitrale locale, nel caso di accoglimento della richiesta.

Contro la decisione del tribunale è possibile presentare ricorso secondo le norme procedurali generali del diritw turco. Tale ricorso sospende l'esecuzione del lodo arbitrale fino alla decisione della corte competente.

27 Unal, loc. cit., p. 71. 